\title{
Lesch-Nyhan Syndrome
}

National Cancer Institute

\section{Source}

National Cancer Institute. Lesch-Nyhan Syndrome. NCI Thesaurus. Code C61255.

An X-linked inherited syndrome caused by mutations in the gene that encodes the enzyme hypoxanthine-guanine phosphoribosyltransferase, resulting in accumulation of uric acid in the body. It affects males and is characterized by neurologic defects, moderate mental retardation, muscle hypotonia, and a tendency for self-mutilation (selfbiting of lips, tongue, and fing ertips). 